\title{
METHODOLOGY OF RESIDUAL STRESS MEASUREMENTS FOR RIVET JOINTS
}

\author{
Jerzy Kaniowski \\ Bartosz Korzeniewski \\ Institute of Aviation, \\ Warsaw, Poland \\ Merja Hakanen \\ Stresstech Oy, Finland
}

\begin{abstract}
The methods and good practice in XRD measurements are presented in this paper. The paper concerns the specimens made of 2024-T3 aluminium alloy plates joint together by rivets. The presented methodology can by divided into two parts: (1) general rules of XRD measurements on 2024-T3 aluminium alloy - choosing the diffraction angle, time of exposure on X-ray radiation, diameter of $X$-ray spot, etc. and (2) rules applied to riveted specimens - geometrical analysis of the specimen and movements of the goniometer which allow to obtain proper results of stress measurement.

Short information about theoretical bases and influence of protective layers on XRD measurement is also included.

In the end of the paper the additional equipment called the slit is presented, which allow to perform measurements on flat and cylindrical surfaces with higher resolution.
\end{abstract}

\section{THEORETICAL BASES}

X-ray diffraction can be used as a non-destructive method to measure residual stress in crystalline materials. The method is based on Bragg's law. Stress changes cause small changes in the lattice distance, which can be measured using x-ray diffraction. Strains and therefore also stresses based on Hooke's law can be calculated from these distance values. Elasticity theory for isotropic solids gives the strain along a specific direction according to

$$
\varepsilon_{\phi \psi}=\frac{1+v}{E} \boldsymbol{\sigma}_{1} \cos ^{2} \phi+\sigma_{2} \sin ^{2} \phi \sin ^{2} \psi-\frac{v}{E} \boldsymbol{\sigma}_{1}+\sigma_{2}-
$$

This equation can be written in the following form $(\varphi=0)$ :

$$
\sigma_{\phi}=\frac{E}{\varangle+v \sin ^{2} \psi}\left(\frac{d_{\psi}-d_{n}}{d_{n}}\right)
$$

For the determination of the stress, $\mathrm{x}$-ray diffraction measurements are done using many tilts and lattice distance is measured and plotted as a function of $\sin ^{2} \psi$. The stress can then be calculated using the slope of this plotted curve. 


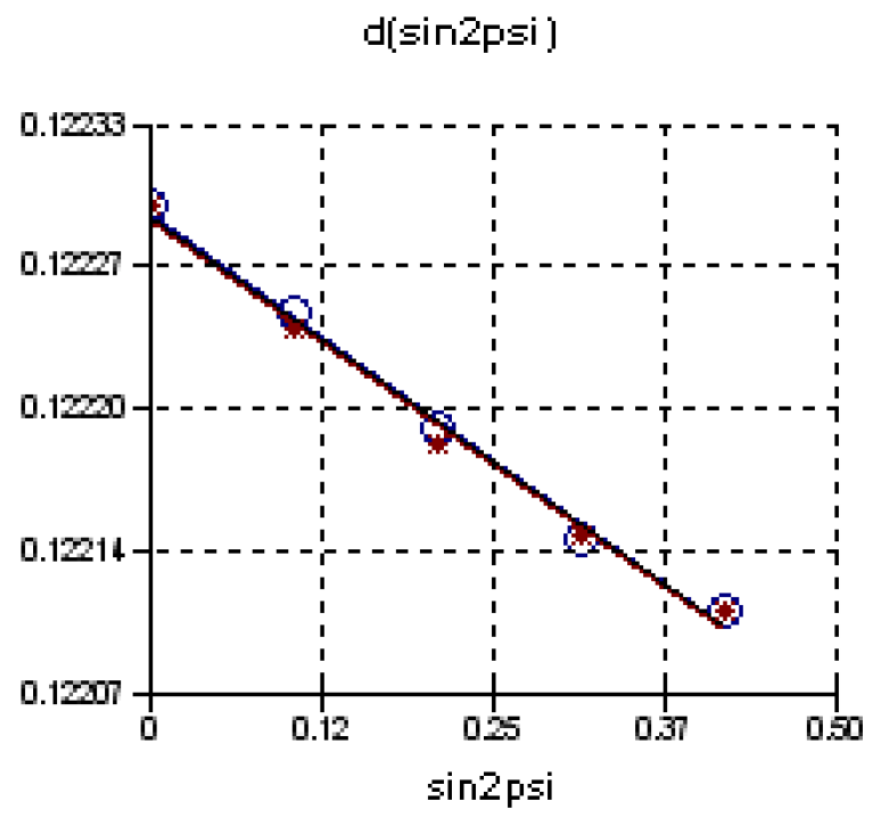

Fig. 1. A d value vs. $\sin ^{2} \psi$ plot measured on riveted aluminium sample

\section{THE INFLUENCE OF PROTECTIVE LAYERS - CLAD AND ANODE}

Many materials absorb strongly $\mathrm{x}$-rays and so the intensity of the x-ray beam decreases exponentially below the sample surface. Also, the diffracted beam originates from the nearby surface and thus the measured residual stress correlates only to this small volume. The influence of the protective layers depends on layer thickness compared to the penetration depth of the radiation. Thickness of the anodizing layer is usually very small compared to x-ray penetration depth, but thickness of certain coatings can be significant.

\section{SELECTION OF MEASUREMENT PARAMETERS}

\subsection{Selection of 2 theta angle}

3.1.1. General rules in selecting the 2 theta angle (e.g. generally, a bigger 2 theta angle is better etc.)

The selection of $2 \theta$ angles is based on Bragg's law. The first x-ray tube should be selected so that the anode material gives Bragg reflection at high $2 \theta$ angles. Differentiating Bragg's law gives:

$$
\frac{\Delta d}{d}=-\Delta \theta \cot \theta
$$

Due to this, the $\Delta d$-value will be too small to be measured at low $2 \theta$ angles. At high $2 \theta$ angles small changes in the lattice distances will give measurable changes in $2 \theta$.

Generally speaking, the highest diffraction angle value should be selected between $2 \theta$ value $125^{\circ}$ and the high $2 \theta$ limit of the diffractometer. It is not recommended to measure using $2 \theta$ values below $125^{\circ}$. 


\subsubsection{Advantages and disadvantages of both angles: $139^{\circ}, 156^{\circ}$}

Crystallographic planes vary in their deformation mechanism and give different strains. Generally, measurements done on different planes are not comparable. If a sample has a large grain size or is textured, it is recommended to use reflection ( $2 \theta$ angles) with the highest multiplicity instead of the highest possible $2 \theta$ angle. For aluminium, when $\mathrm{CrK} \alpha$ radiation is used, the highest multiplicity comes from hkl plane $311,2 \theta$ angle $139.3^{\circ}$ (Multiplicity factor for (311) plane is 24 and for (222) plane is 8). Using other reflections may increase oscillation and scatter in the $\sin ^{2} \psi$ graphs.

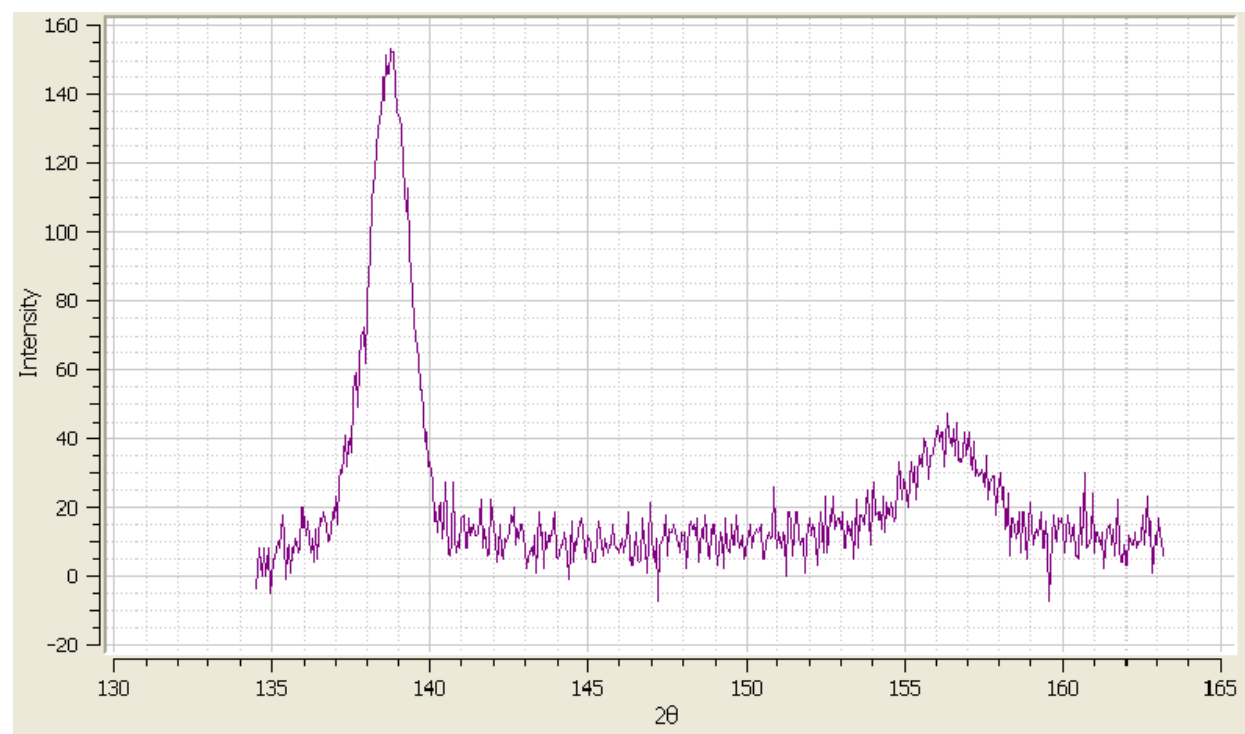

Fig. 2. Diffraction peaks measured on riveted aluminium sample. This shows that the reflection from (311) plane at $2 \theta$ angle $139,3^{\circ}$ is stronger than the reflection from (222) plane at $2 \theta$ angle $156,7^{\circ}$
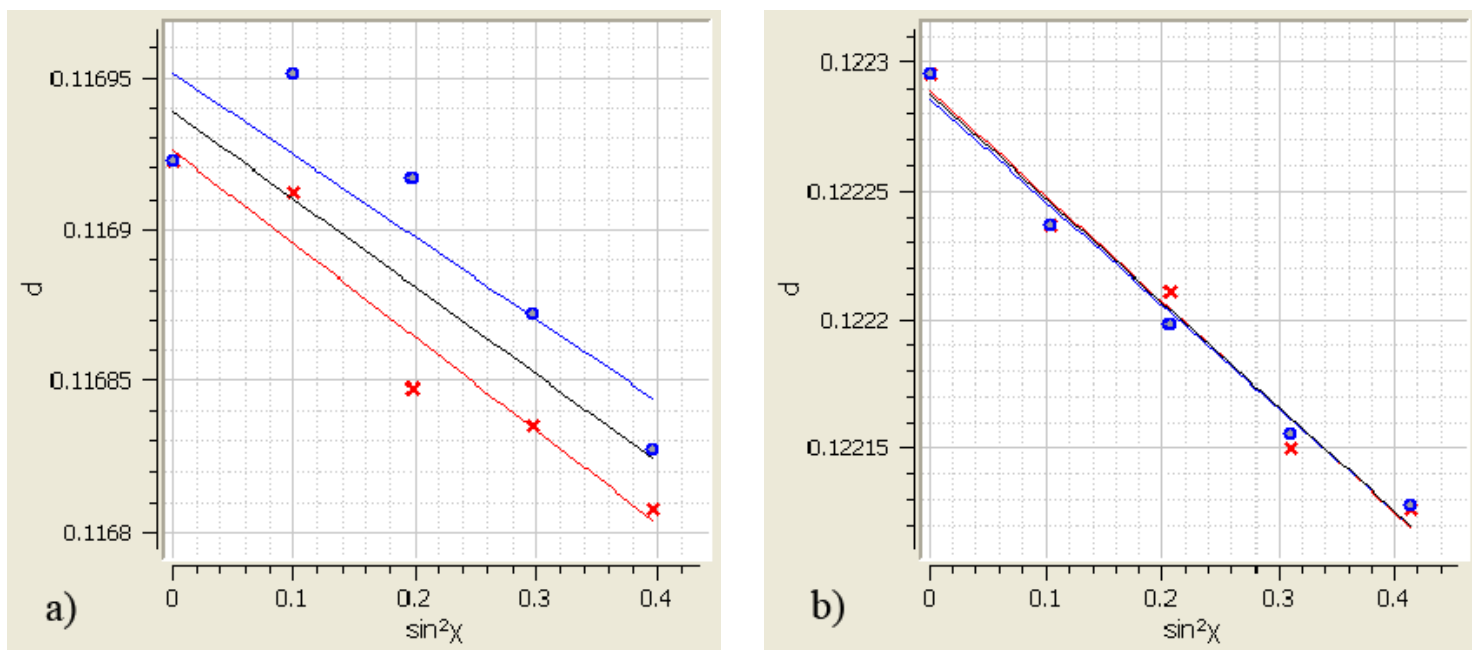

Fig. 3. A d value vs. $\sin ^{2} \psi$ plots measured using $156,7^{\circ}\left(a-\right.$ left graph) and $139,3^{\circ}(b-$ right graph $)$ measured on riveted aluminium samples

3.1.3. Conclusion for 2024-T3 aluminium alloy sheet

Diffraction angle $139,3^{\circ}$ was selected for measurement. 


\subsection{Selection of the collimator diameter}

The collimator size should be selected so that the out coming x-ray beam covers the measurement area. For round samples, in measurement to the hoop direction, it is recommended to use quarter of the sample radius as the collimator size.

Because the stress distribution is changing much in a small area near the rivet, it is recommended to use as small collimator diameter as possible in order to obtain the best resolution: $0.5 \mathrm{~mm}$ and $0.8 \mathrm{~mm}$ collimators were used.

\subsection{Selection of time of exposure}

In general, exposure time should be long enough to obtain a well-defined peak, with G2R diffractometer and standard detectors this usually means a peak with intensity of more than 40 units. A good method to determine the optimal exposure time is to continue measurements using increasing exposure time until no more improvements in the results are obtained. If the sample is textured or has large grain size, along with exposure time, oscillations should be optimized.

Exposure time can be reduced by modifying the measurement method. Using full power (30 $\mathrm{kV}, 9 \mathrm{~mA}$ ) will reduce exposure time. The diffractometer can be modified by replacing standard detectors with long detectors. This alone will reduce exposure time by about $30 \%$. Using long detectors makes it also possible to use the $\varnothing 0.5 \mathrm{~mm}$ collimator. Standard detectors do not give reasonable results. It was observed that cooling of the detectors will not improve the quality of the intensity data. If the XTronic software with the diffractometer is used, subtraction of the dark current can be made only once at the beginning of each measurement direction, which reduces exposure time by about $50 \%$.

\subsection{Selection of $\Psi$ tilts (maximum value of $\Psi$ tilt, number of tilts, and value of $\Psi$ oscillations)}

When measuring stress, it is recommended to use at least 4 tilt angles on both negative and positive tilt $\Psi$ angles, using tilt angles between $0^{\circ}-45^{\circ}$. If the sample is textured, increasing the number of tilt angles might improve the stress results. In that case, also oscillation of tilt angles might improve the results, although this will give some uncertainty of the $\Psi$ angle in the $\mathrm{d}\left(\sin ^{2} \Psi\right)$ graphs. Maximum number of tilts in the X3000 software is 10 and maximum tilt oscillation is $6^{\circ}$.

\subsection{Selection of $\Phi$ oscillations (maximum value of $\Phi$ oscillations, number of oscillations)}

Oscillation of the $\Phi$ angle might improve stress results when textured and large grain sized samples are involved. Oscillations of up to $\pm 5^{\circ}$ can be made without significantly affecting the accuracy of stress in terms of measurement direction. It is not recommended to use more than $\pm 10^{\circ}$ oscillation. Increasing the number of the oscillation steps will improve the measurement and will also increase the total exposure time.

\section{LOCATION OF MEASUREMENT POINTS}

4.1. Method for initial determination of the shape of stress versus radial distance function and location of the peak (extreme) stress point-for tangential stress (two tilt method, graph with curves obtained with two tilt and standard method).

A method to find rough estimate of the stress distribution along the measurement path was proposed. The measurement using only tilts 0 and $-45^{\circ} / 45^{\circ}$ angles and otherwise the same parameters as in standard measurements is more quickly made and gives an estimate of the stress. After this test, the measurement points can be selected for a closer study. In order to further decrease the measurement time in the two tilt method, exposure time can be reduced significantly based on the results shown in Fig 4. 
In the graph below, stress measurement has been made using the standard method and afterwards, two tilt method results have been recalculated by disabling results from other tilts except $0^{\circ}$ and $\pm 45^{\circ}$.

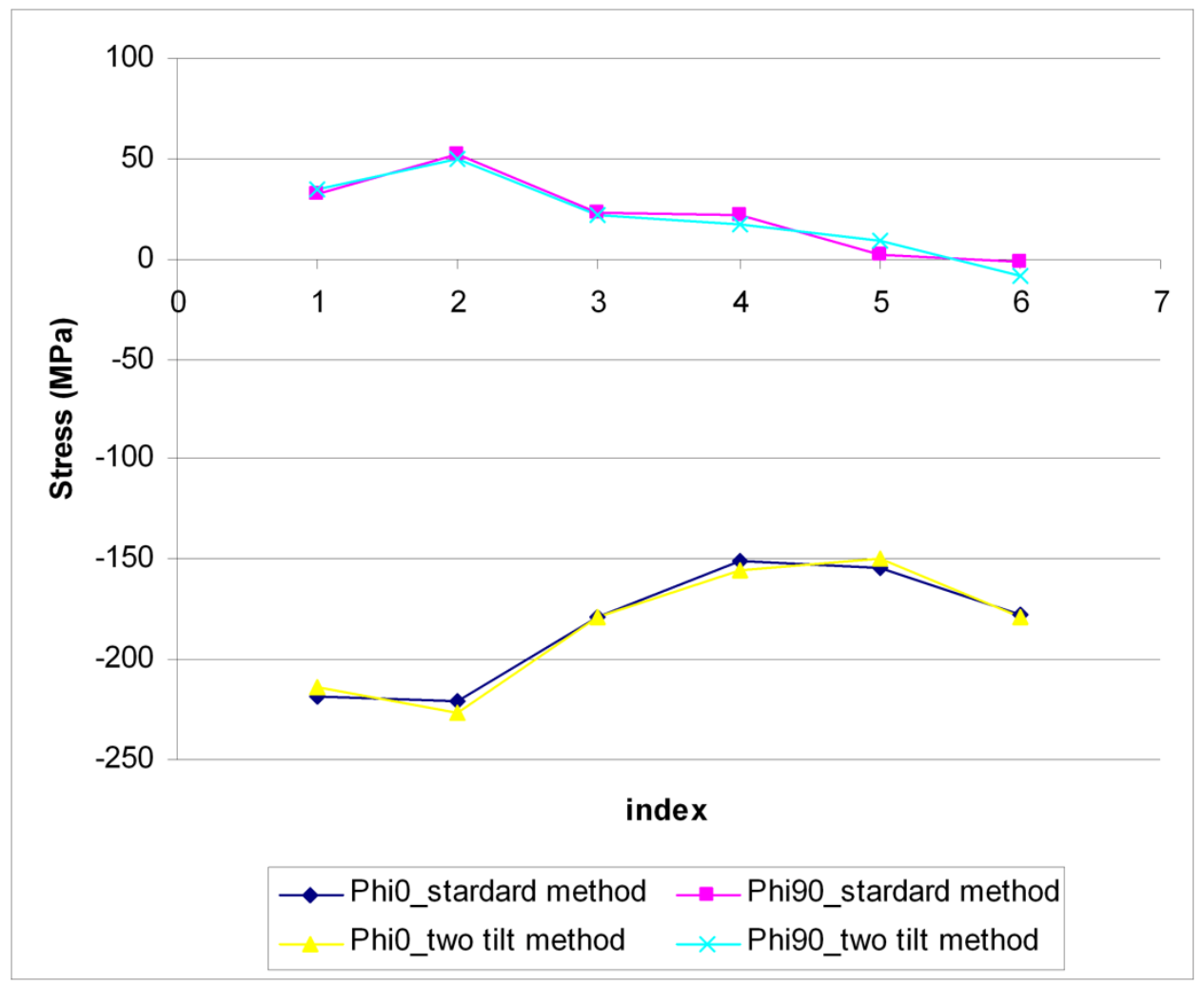

Fig. 4. Example of two tilts method vs. standard method

\subsection{Determination of measurement points location on measurement path}

Measurement points can be selected according to the results of the two tilt measurement test.

\subsection{Position of measuring paths}

The measurements should be made on both sides of the specimen (fig. 5):

- $\quad$ upper side - manufactured head side and,

- $\quad$ lower side - driven head side,

along paths parallel to the longer edge of the specimen (shown on the specimen, fig. 6).

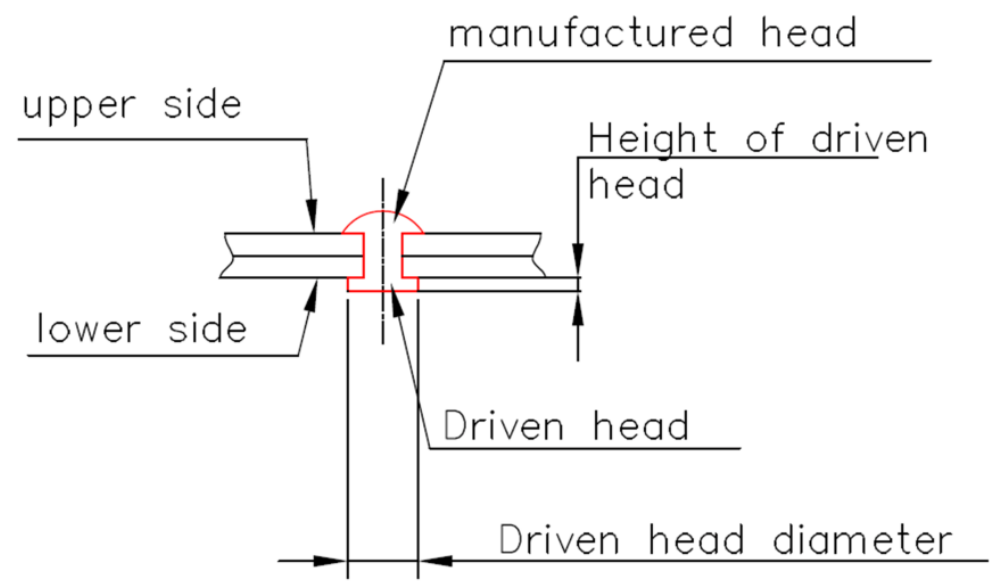

Fig. 5. Used designation 


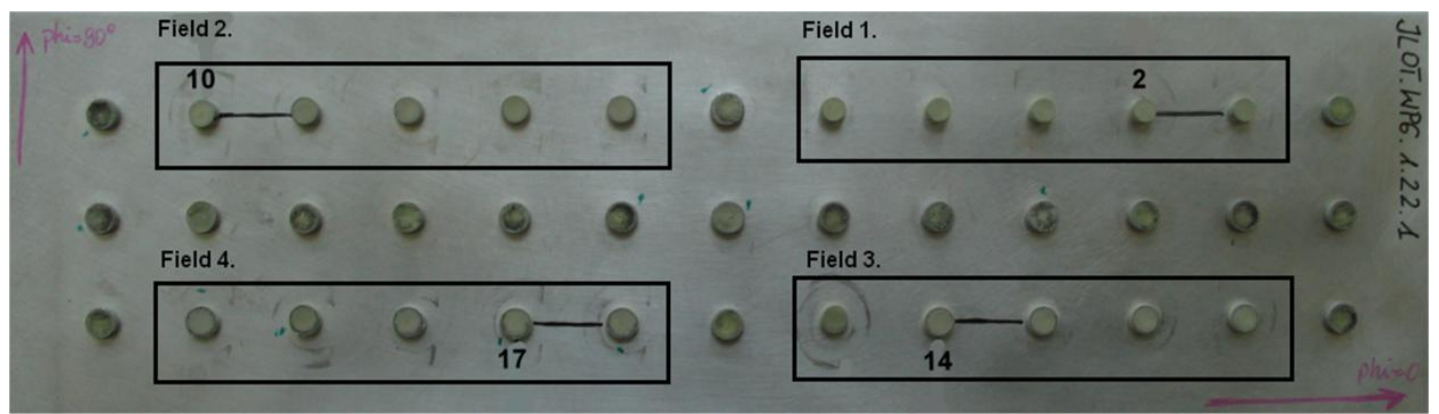

Fig. 6. Measurement paths drawn on specimen (view from the driven head side - lower side - that's why fields 1. and 3. are on the other side than in fig. 1.)

On every measurement path, measurements should be made at 7 points. The first measurement point on every path should be defined by touching the rivet head (or upset head) by the collimator (in WP6.1.2.1 by touching the edge of the countersink), fig. 7, and 8.

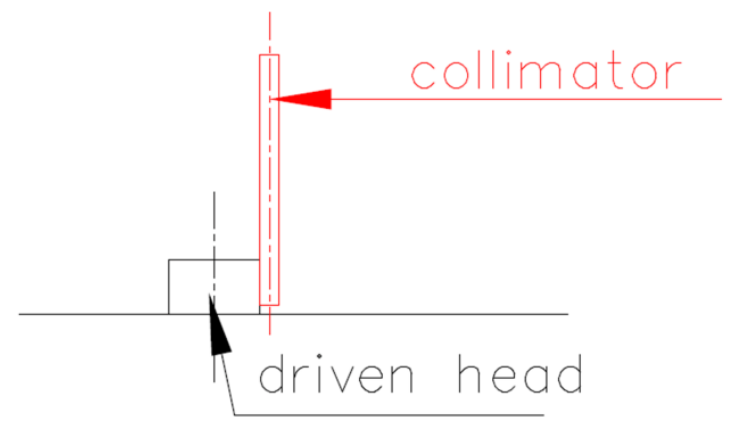

Fig. 7. Determination of first measurement point - by touching the driven head.
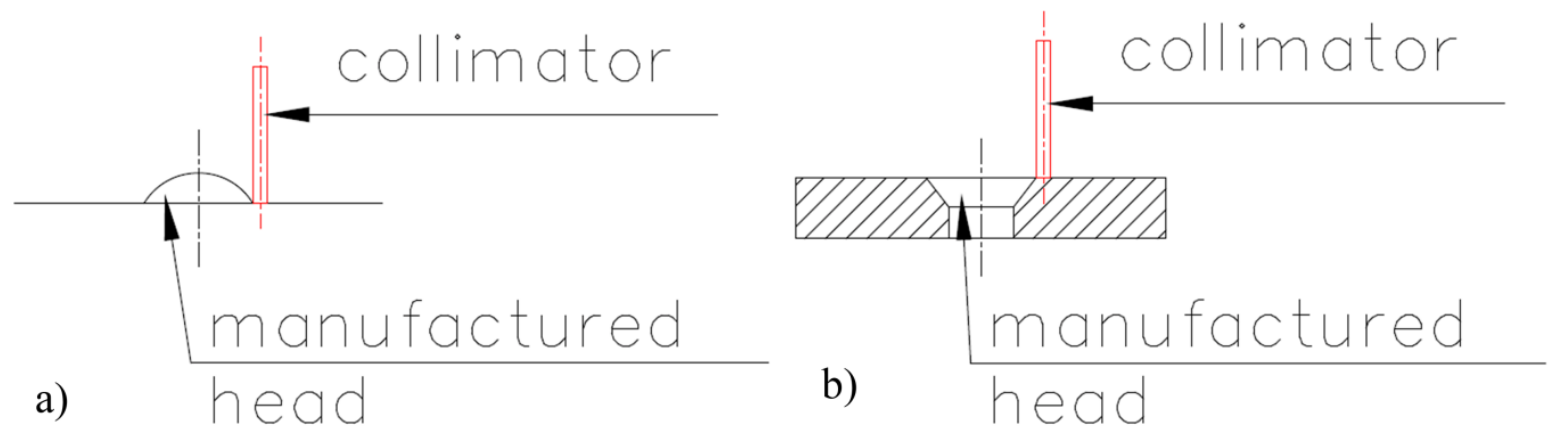

Fig. 8. Determination of first measurement point - by touching the manufactured head a) for a rivet with a protruding head, b) for a rivet with a conical head (countersunk rivet)

\section{GEOMETRICAL ANALYSIS OF THE MANUFACTURED HEAD (DRIVEN HEAD) IMPACT ON RADIAL STRESS MEASUREMENT.}

One should always bear in mind that, for certain psi angles, the x-ray beam could be disrupted by the manufactured head (driven head). This is why the geometrical analysis is obligatory as it uncovers which tilts are acceptable. For the measurement points in intimate surroundings of the driven head (manufactured head) during tilt of about $\boldsymbol{\Psi}_{\mathbf{1}}$ (psi 1) toward the driven head (manufactured head) (fig. 9), the X-ray beam collides with the driven head (manufactured head) without reaching the measurement point. This disruption is called a driven head (manufactured head) shadow. 


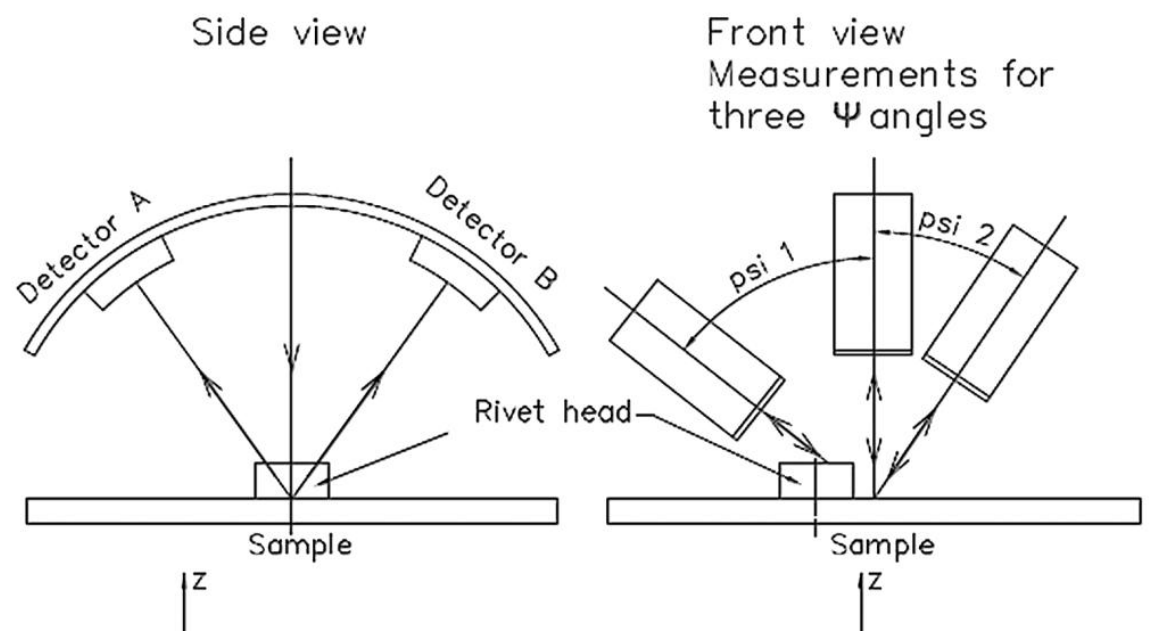

Fig. 9. Analysis of a driven head shadow

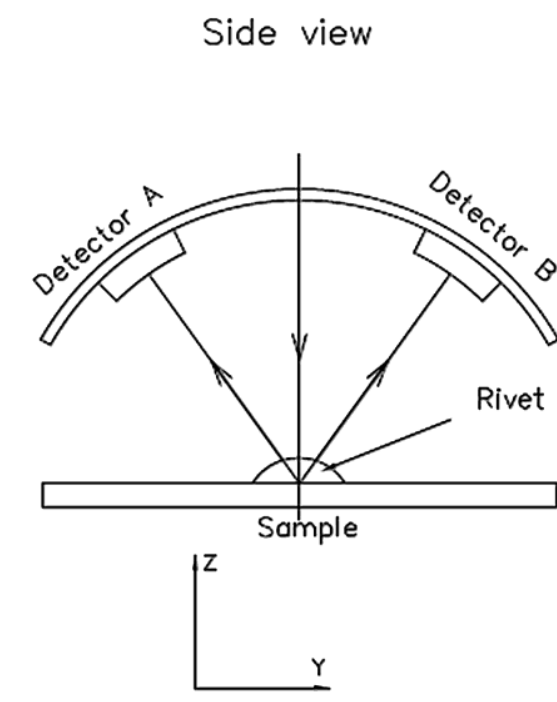

Front view
Measurements for
three $\Psi$ angles

Fig. 10. Analysis of a manufactured head shadow - radial stress measurement

For measurement points in intimate surroundings of the driven head (manufactured head), geometrical analysis should reveal the collision of the $\mathrm{x}$-ray beam with the driven head (manufactured head).
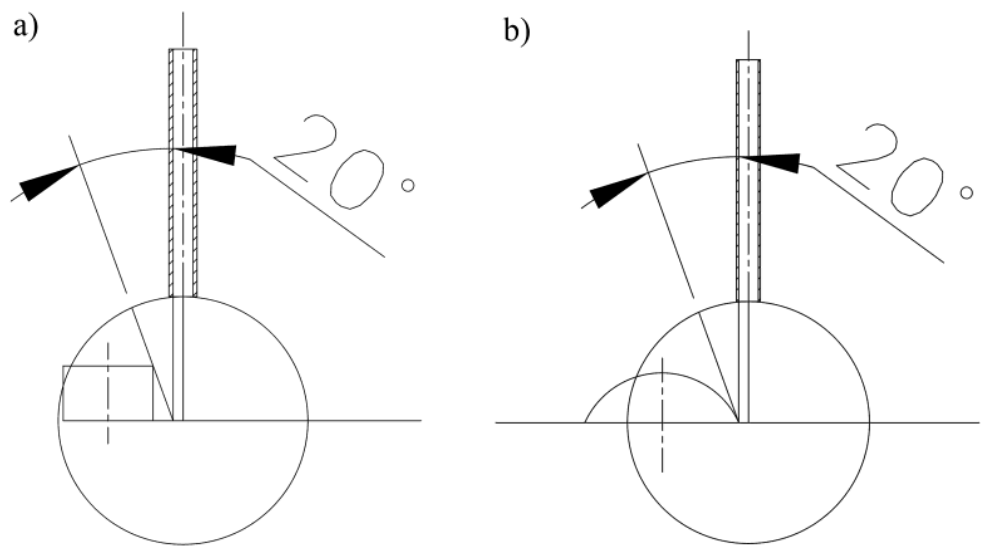

Fig. 11. Designation of boundary $\psi$ anglein which $x$-ray beam generatrix touches the a) driven head, b) manufactured head 
Another important consideration is the possibility of collision between the collimator and driven head (manufactured head). For a calibrated distance from specimen "d" at measurement points in intimate surroundings of the driven head (manufactured head) during tilt of about $\boldsymbol{\Psi}$ (psi 1) toward the driven head (manufactured head), the collimator could touch the driven head (manufactured head), fig. 13. The angle at which the collision occurs is called $\boldsymbol{\Psi}_{\text {collision. }}$. For the points in the measurement program (path), one should only accept these where $\boldsymbol{\Psi}<\boldsymbol{\Psi}_{\text {collision }}$.

The analysis of the manufactured head impact on radial stress measurements should also be made this way.

\section{GEOMETRICAL ANALYSIS OF THE MANUFACTURED HEAD (DRIVEN HEAD) IMPACT ON CIRCUMFERENTIAL STRESS MEASUREMENTS.}

One should always bear in mind that in intimate surroundings of the driven head (manufactured head), the diffracted x-ray beam collides with the driven head (manufactured head) and does not reach one of the detectors (in Fig. 12 - detector A). To eliminate this problem the signal only from the uncovered detector is taken into account when calculating the circumferential stress.

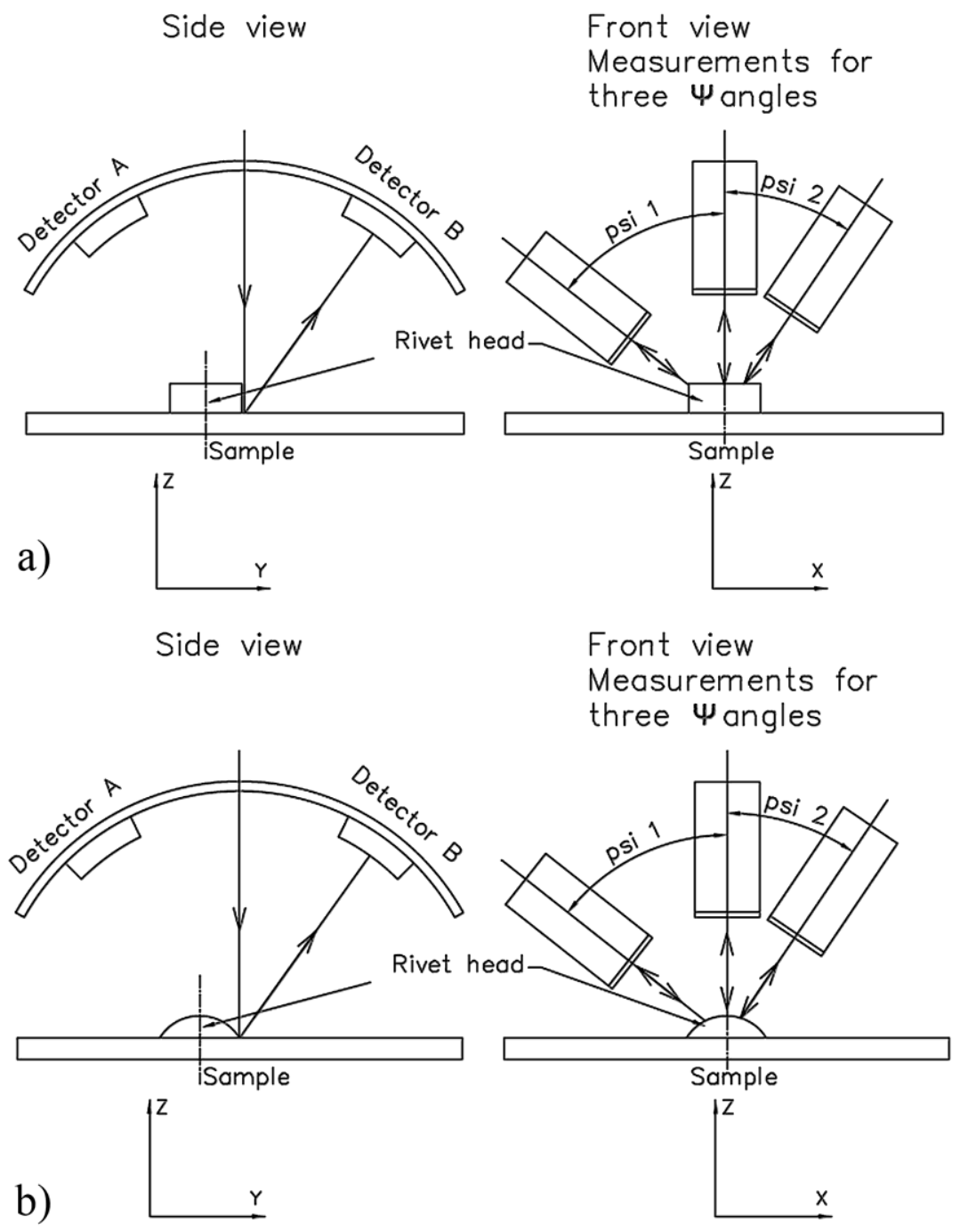

Fig. 12. Analysis of a driven head (manufactured head) shadow - circumferential stress measurement, a) by driven head, b) by protruding head. 
The analysis of the manufactured head impact on circumferential stress measurements should also be made this way.

\section{MEASUREMENT PARAMETERS TABLE}

Measurement parameters should be selected according to point 3 of this methodology and inserted in the table shown below.

These parameters should be constant for all measurements on particular (here WP6.1) specimen series.

Tab. 1.

\begin{tabular}{|c|c|c|}
\hline & Parameters & Values \\
\hline 1. Data for calculation: & $\begin{array}{l}\text { 2theta } \\
\text { Background subtraction } \\
\text { Detectors } \\
\text { Peak limit } \\
\text { Peak shift }\end{array}$ & $\begin{array}{l}\text { Calibrated } \\
\text { Linear } \\
\text { A and B (the changing parameter, see the } § 5 \text { ) } \\
\text { No } \\
\text { Cross corr. }\end{array}$ \\
\hline 2. Calibrated distance: & d & $\begin{array}{l}4.46 \mathrm{~mm} \text { (the changing parameter, dependent } \\
\text { from result of diffractometer calibration) }\end{array}$ \\
\hline 3. Material data: & $\begin{array}{l}\text { Material } \\
\text { Young module } \mathbf{E} \\
\text { Absorption coefficient } \boldsymbol{\mu} \\
\text { Poisson's ratio } \boldsymbol{v}\end{array}$ & $\begin{array}{l}\mathrm{Al}\left(139,3^{\circ} \text { or } 156,7^{\circ}\right) \\
70600 \mathrm{MPa} \\
42.71 / \mathrm{mm} \\
0.345\end{array}$ \\
\hline $\begin{array}{l}\text { 4. Measurement } \\
\text { parameters: }\end{array}$ & $\begin{array}{l}2 \Theta / h k l \\
\text { Time of exposition } \\
\text { Method of measurement } \\
\Phi \text { angles } \\
\boldsymbol{\Phi} \text { oscillations } \\
\text { Angles of boundary } \boldsymbol{\Psi} \\
\text { Angles and oscillations number of } \boldsymbol{\Psi} \\
\text { Radiation }\end{array}$ & $\begin{array}{l}139,3^{\circ} / 311 \text { or } 156,7^{\circ} / 222 \\
\max 120 \mathrm{~s} \text { (may vary) } \\
\text { Psi } \\
0^{\circ} ; 90^{\circ} \\
10^{\circ} / 5 \\
-39^{\circ} /+39^{\circ} \\
\pm 6^{\circ} ; 5 / 5 \\
\mathrm{CrKa}\end{array}$ \\
\hline 5. Collimator: & Diameter & 0,5 or $0,8 \mathrm{~mm}$ \\
\hline
\end{tabular}

Boundary $\boldsymbol{\Psi}$ angles should be selected with information from point 4 . For the measurement points in intimate surroundings of the driven head (manufactured head), for which it was necessary to eliminate more than $2 \Psi \boldsymbol{\Psi}$ angles, it is obligatory to eliminate all $\boldsymbol{\Psi}$ tilts to that side (the side where it was obligatory to eliminate tilts) - also due to collimator collision with the driven head (manufactured head) the safest choice is to use tilts to only one side. This way there are two radial stress measurement programs for one measurement path within one project:

- One - for points near the driven head (manufactured head), the tilts are limited to only one side. - Second - tilts are not limited $\left(-39^{\circ} /+39^{\circ}\right)$.

This way it is possible to bypass two issues - the driven head (manufactured head) shadow and the collimator collision.

Attention: When x-ray spot is entering the driven head (manufactured head) it is obligatory to increase the number of $\boldsymbol{\Psi}$ tilts.

\section{FIXING THE SPECIMEN TO X-Y TABLE}

Fixing the specimen to $x-y$ table is made with fixing grips - Fig. 13. After the riveting process the specimen are not perfectly flat. When the specimens are placed on grips they will probably swing a little. There should be a central swing position found and in this position swing should be 
compensated by use of gaskets of adequate thickness. After this procedure, holding screws should be twisted. The screws should be twisted only by hand.

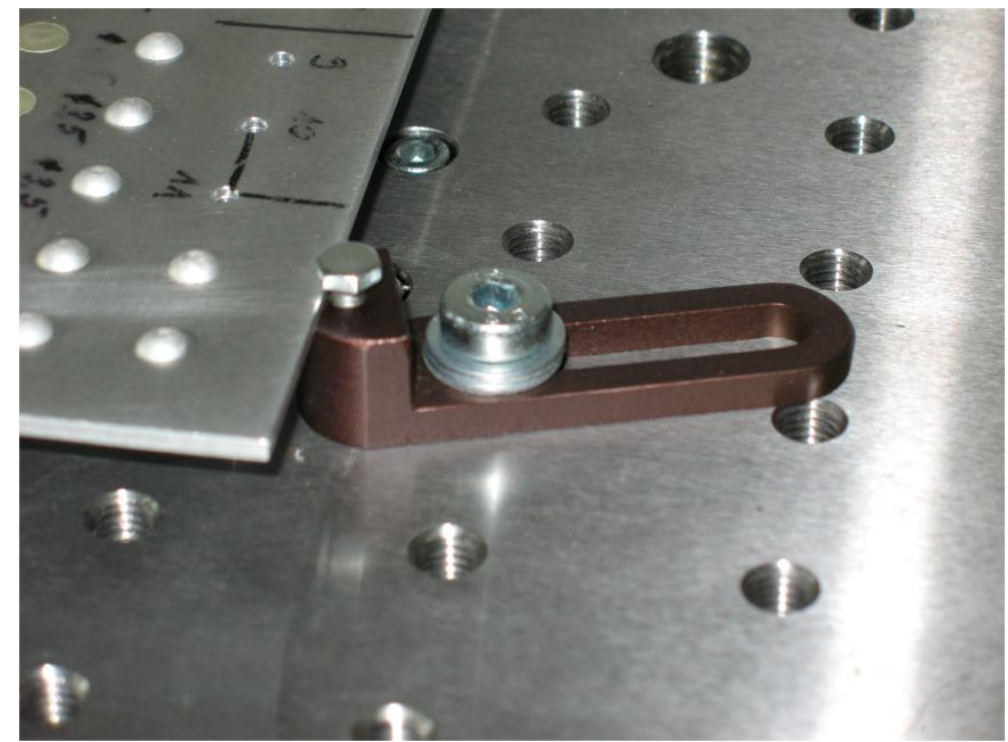

Fig. 13. The method of fixing the specimen to $x$-y table

\section{USAGE OF THE SLIT}

The slit will determine precisely the measurement area and volume, fig. 14 and 15 . If measurement is made without a slit, the $\mathrm{x}$-ray beam radius at tilt angles $\pm 45^{\circ}$ will be $\sqrt{2}$ times bigger compared to radius at tilt angle $0^{\circ}$. Also the penetration depth when using a slit will be more constant at different tilt angles. This makes the measurement volume ill defined. Material for the slit is selected so that no diffraction peaks can appear in the selected $2 \theta$ range, for example $\mathrm{Pb}$. The slit should be thin and touch the sample surface so that its effect on the collimator distance would be minimal.

The slit is put over the sample. The sample moves under the slit. The slit is so thin that you can touch it by the collimator and it does not affect measurement results. The slit is made from lead $(\mathrm{Pb})$. You can adjust the size of the slit for use with several collimator diameters.

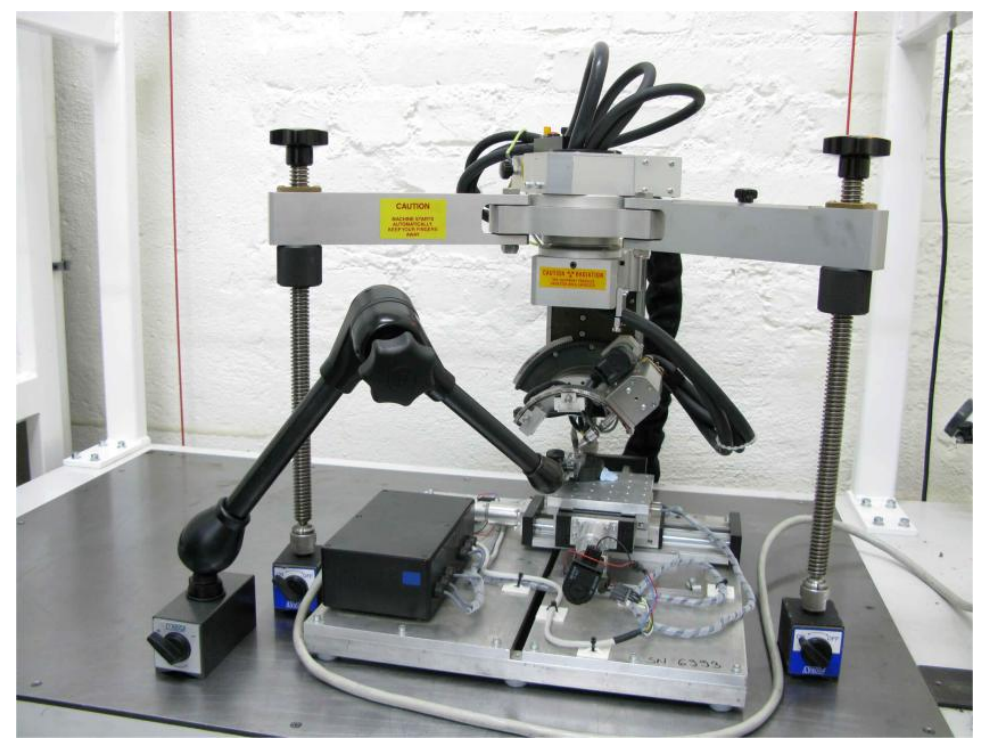

Fig. 14. Device with lead slit attached 


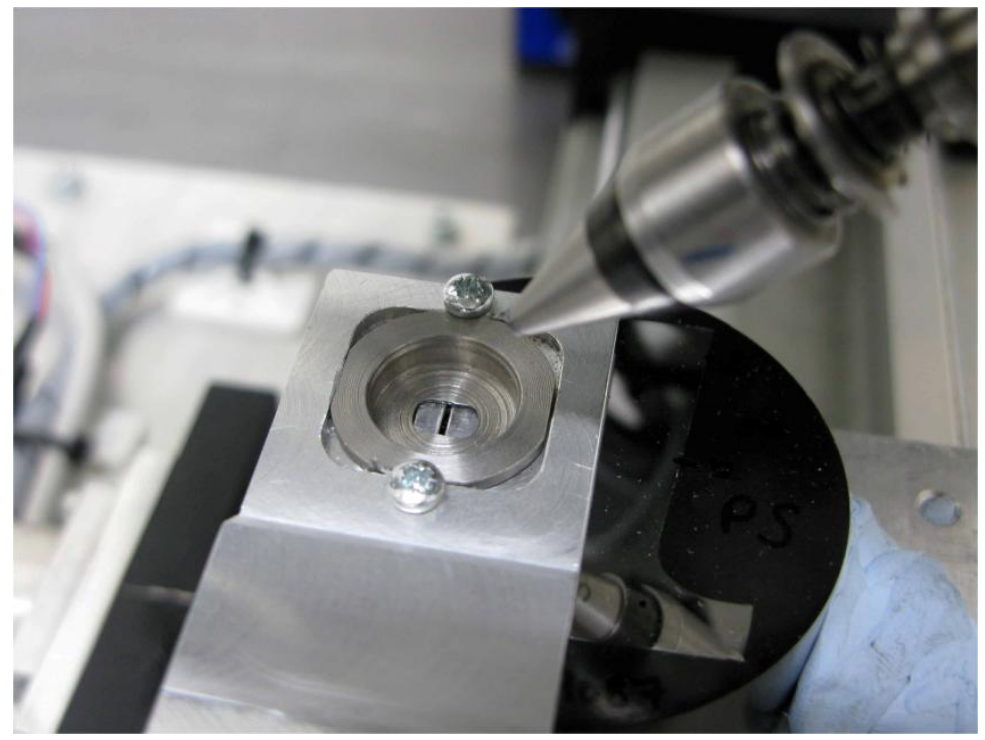

Fig. 15. Lead slit

\section{APPOINTING THE INCREASE OF STRESSES BY RIVETING}

In the zone where during riveting no plastic deformation was introduced, the stress increase, associated with riveting, could be determined by simple subtraction of the stress before riveting from the stress measured after riveting.

$$
\sigma=\sigma_{\text {after riveting }}-\sigma_{\text {after drilling of hole }}
$$

\section{ACKNOWLEDGEMENT}

The authors would like to thank Dr A. Wojtas for his useful comments and discussions.

\section{REFERENCES}

[1] XSTRESS 3000 G2/G2R. X-ray Stress Analyzer. Technical Manual V. 1.1 b. Stresstech Oy., 2007.

[2] XSTRESS 3000 G2/G2R. X-ray Stress Analyzer. Software User's Guide X3000 V. 1.22d. Stresstech Oy., 2007.

[3] M.E. Fitzpatric, A.T. Fry, P. Holdway, F.A. Kandil, J. Shackleton, L. Suominen, "Determination of Residual Stresses by X-Ray Diffraction - Issue 2", A National Measurement Good Practice Guide No.52, National Physical Laboratory, 2005. 